\title{
Binary Ethylenimine Inactivated Japanese Encephalitis Virus Antigen Reveals Hemagglutination
}

\author{
Dong-Kun Yang*, Ha-Hyun Kim, Jin-Ju Nah, Kyung-Woo Lee, Jae-Young Song \\ Animal, Plant and Fishery Quarantine Inspection Agency (MIFAFF), Anyang, Korea \\ Email: *yangdk@korea.kr
}

Received June 28, 2012; revised July 23, 2012; accepted July 31, 2012

\begin{abstract}
Severe climate change and global warming may impact significantly on vector-borne disease including Japanese encephalitis (JE) infection in human and animals. Thus, veterinary authority requires large quantity of diagnostic tools to survey vector-borne diseases. New producing method having a relation with JE antigen is needed to substitute conventional sucrose-acetone extraction method using suckling mouse. So, we developed new manufacturing method using polyethylene glycol (PEG) precipitation. Japanese encephalitis virus (JEV) was propagated in roller bottle containing Vero cell and inactivated with two kinds of inactivating reagents. Viability of the supernatant of bulk containing antigen was checked using Vero cell after inactivation. The supernatant did not show hemagglutination (HA) activity with goose erythrocytes. The antigen inactivated by binary ethylenimine (BEI) and concentrated by PEG precipitation method was found to be $2048 \mathrm{HA}$, but the antigen inactivated by $0.3 \%$ formaldehyde solution and concentrated by PEG precipitation method did not show HA titer. The antigen prepared from mice brain using sucrose-acetone extraction method showed $256 \mathrm{HA}$ titer. This BEI inactivation method does not evoke animal welfare problem and can replace the conventional method that required biological hazardous reagents and suckling mice in preparing HA antigen. This new BEI inactivation method was safe in producing HA antigen against JEV in laboratory and can reduce environmental contamination of acetone.
\end{abstract}

Keywords: Hemagglutination; JEV; BEI Inactivation

\section{Introduction}

Climate change through recent global warming and extreme weather may have significantly impacted the transmission of vector-borne diseases such as Japanese encephalitis virus (JEV), West Nile virus (WNV), and tick-borne encephalitis virus (TBEV), as rapid weather and climate changes can directly or indirectly affect migratory birds and mosquitoes. Transmission of JEV is well kept in a mainly rural zoonotic cycle between mosquitoes and wild birds including egrets and herons $[1,2]$.

Approximately 67,900 Japanese encephalitis (JE) cases occur annually in Asian countries and about $50 \%$ of JE cases occur in China [3]. JE outbreak occurs in children aged 0 - 14 years. Most infections in human are inapparent or produce a mild systemic illness with fever, headache or asceptic meningitis. Inflammation of the brain, spinal cord and meninges can cause stupor, tremors, convulsions (especially in infants), spastic paralysis, coma and death [4]. Patients infected with JE show hearing and speech impaired judgment in recovery period. JEV can also lead to infection in several animals such as

"Corresponding author. reptiles, pigs, horses, chickens, breeding birds such as herons. In particular, livestock pigs infected with JEV can amplify the JEV in body and are known as an amplifying host [5]. Fattening pigs bitten by mosquitoes holding JEV show viremia for a short period of time with subclinical signs. However, pregnant sows with JEV infection cause still birth, mummified fetus, weaken piglets showing neurological symptoms. Boars are also infected with JEV that penetrates boar's genital organ and make the boar reduce a number of sperm and deform sperms resulting in dropping pregnancy rate. Horse infected with JEV naturally shows lack of appetite, fever, neurological symptoms and pass away in the long run [6]. Veterinary authorities in South Korea have been tried to immunize livestock to block JE epidemic as well as to block the influx and to predict the disease in pigs. Since 1990, pigs being raised on farm have been vaccinated against JE. And since 2001, sero-surverveillance for JEV has been carried out over 9000 pigs every year.

When the blood-agglutinating viruses such as porcine parvovirus (PPV), encephalomyocarditis virus (EMCV) are propagated in cell culture systems and used as a hemagglutinating antigen, they still possess hemagglutinat- 
ing activity. However, when JEV is cultivated and propagated in cell culture system, the supernatant of the JEV does not have hemagglutinating activity. Therefore, hemagglutinating antigens prepared from suckling mice brains infected with JEV using the sucrose-acetone extracting method has been used in checking immune status of JEV in pigs. However, the sucrose-acetone extracting method is biologically hazardous in the process to manufacturers and has caused a debate about animal welfare. Thus, new method to produce JEV antigen without using suckling mice has been demanded in laboratory. In this study, we report a new method to make highly concentrated JEV HA antigen using cell culture system.

\section{Material and Methods}

\subsection{Propagation of Virus and Cells}

The strain of JEV used as an antigen for the hemmagglutination inhibition (HI) test was KV1899. This strain isolated from Korean pig blood in 1999 was propagated using Vero cells cultured in $\alpha$-minimum essential medium (MEM; Gibco BRL, USA) supplemented with antibiotics $(100 \mathrm{IU} / \mathrm{mL}$ penicillin and $100 \mu \mathrm{g} / \mathrm{mL}$ streptomycin), an antimycotic $(0.25 \mu \mathrm{g} / \mathrm{mL}$ amphotericin B), and $5 \%$ fetal bovine serum (FBS; Gibco BRL). After inoculation, when $90 \%$ of cytopathic (CPE) effects in cells were shown, the roller bottles were frozen at $-70^{\circ} \mathrm{C}$ and thawed three times.

\subsection{Inactivation of Antigen}

The JEV was inactivated with binary ethylenimine (BEI) by method of Larghi et al. [7]. In brief, BEI was prepared from 2\% 2-bromo-ethylamine hydrobromide (2-BEA, Sigma, USA) in solution of $0.2 \mathrm{~N} \mathrm{NaOH}$, and the solution was treated in water bath at $37^{\circ} \mathrm{C}$ for $1 \mathrm{hr}$, and then was adjusted to $0.1 \mathrm{M}$ BEI. The final concentration of BEI was adjusted to $0.001 \mathrm{M}$ of bulk and $\mathrm{pH}$ of bulks also was adjusted to 8.0 with $1 \mathrm{~N} \mathrm{NaOH}$. Inactivation was done at $37^{\circ} \mathrm{C}$ for $10 \mathrm{hrs}$. As another inactivation method, $37 \%$ of formaldehyde solution (Junsei chemicals Ltd., Japan) was added to viral suspensions to make final formaldehyde concentration equal to $0.3 \%$ [8]. Inactivation of the JEV by formaldehyde was also done at $37^{\circ} \mathrm{C}$ for $10 \mathrm{hrs}$. Vero cells were employed to check whether the viruses were inactivated or not. After checking the inactivation of viruses, bulks were used for preparation of high concentrated HA antigen.

\subsection{Polyethylene Glycol Precipitation}

The inactivated JEV was centrifuged at $3000 \times \mathrm{g}$ to remove cell debris and concentration by polyethylene glycol (PEG) precipitation was carried out essentially as described by Lewis and Metcalf (1988), with some modi- fication [9]. Sodium chloride was added to obtain a final concentration of $0.5 \mathrm{M}$ and PEG (Sigma, USA, MW 8000 ) was added to a final concentration of $8 \%$. The antigen was placed at $4^{\circ} \mathrm{C}$ for $10 \mathrm{hrs}$ for the precipitation of virus and then centrifuged at $10,000 \times \mathrm{g}$ for 30 minutes. The supernatant medium was poured off leaving the centrifuged tubes inverted until the medium has been removed. The precipitate was suspended in $4 \%$ borate buffered saline (BBS: $\mathrm{pH}$ 9.0) or phosphate buffered saline (PBS: $\mathrm{pH}$ 7.2) using $1 \%$ of the original volume. The high concentrated antigen was stored at $-70^{\circ} \mathrm{C}$.

\subsection{Preparation of Viral Antigen with Mice Brain}

The mice inoculation experiment described in this study had been approved by the Animal Ethics Committee of our institute (Animal Ethics number : QIA-AEC-2010-44) antigen using a sucrose - acetone extraction method was also prepared from the brains of suckling mice infected with the Korean isolate of JEV strain KV1899 [10,11]. Briefly, JEV was inoculated into the brain of mice aging 3 to 4 days. When the inoculated mice began to show signs of paralysis, the mice were euthanized by $\mathrm{CO}_{2}$ and frozen at $-70^{\circ} \mathrm{C}$. The infected suckling mice-mouse brain was homogenized with 5 volumes of $8.5 \%$ sucrose solution and the homogenate was added to 20 volumes of chilled acetone. After shaking vigorously, the milky supernatant was discarded and an equal volume of acetone was added to the flask. This preparation was incubated for $1 \mathrm{hr}$ at $4^{\circ} \mathrm{C}$ to dehydrate the sediment. The supernatant was discarded and the sediment was dried using a vacuum pump. The dried antigen was suspended in saline. After centrifugation for $10 \mathrm{~min}$ at $8000 \times \mathrm{g}$, the supernatant was used as antigen for hemagglutination assay.

\subsection{Hemagglutination Assay (HA) Test}

An HA test to check HA titer of concentrated JEV antigen was performed in 96-well microplates, using slightly modified standard methods [10]. In brief, $50 \mu \mathrm{L}$ of concentrated antigen was diluted with $0.4 \%$ borate saline solution. After dilution, $50 \mu \mathrm{L}$ of $0.33 \%$ goose erythrocytes were added, and the microplates were incubated at room temperature for 30 minutes. The HA titer was expressed as the reciprocal of the highest dilution of antigen showing complete hemagglutination.

\section{Results}

The JEV propagated in roller bottle containing Vero cell was inactivated with two kinds of inactivates. After inactivation, some part of bulk was dialyzed in phosphate buffer. Both supernatants inactivated with both $0.3 \%$ formaldehyde and $0.001 \mathrm{M}$ BEI did not exhibit specific 
CPE in Vero cells, respectively. The supernatants did not reveal HA activity with goose erythrocytes (Table 1). The antigen inactivated with $0.001 \mathrm{M} \mathrm{BEI}$ and concentrated by PEG precipitation method showed 2048 HA titer, but the antigen inactivated by $0.3 \%$ formaldehyde solution and concentrated by PEG precipitation method did not show HA titer (Table 1). The concentrated antigen suspended in PBS at pH 7.2 did not show HA activity with goose erythrocytes, but the antigen suspended in PBS at pH 9.0 showed 2048 HA titer (Table 2). The antigen by BEI inactivation method was titrated with goose erythrocytes and showed 2048 HA titer. The antigen prepared from suckling mice brain using sucrose-acetone extraction method showed 256 HA titer (Figure 1).

\section{Discussion}

Since children vaccination program was introduced in Korea in 1960's, JE vaccine has been inoculated into most children. The JE vaccine program results in leading low JE case in human [12]. However, the recent climate change affects JEV infection rate in the human population as $45 \mathrm{JEV}$ cases were reported by the Korean Center for

Table 1. HA titer of JEV antigen according to inactivating agents.

\begin{tabular}{ccccc}
\hline & \multicolumn{2}{c}{ Supernatant } & \multicolumn{3}{c}{ Concentrated antigen } \\
\hline Inactivating agent & Formaldehyde & BEI $^{*}$ & Formaldehyde & BEI \\
\hline HA titer & $<2$ & $<2$ & $<2$ & 2048 \\
\hline
\end{tabular}

*Binary ethylaminimine.

Table 2. Optimal buffer conditions for BEI method.

\begin{tabular}{ccc}
\hline & \multicolumn{2}{c}{ Concentrated antigen } \\
\hline Diluent & $\begin{array}{c}\text { Phosphate buffered } \\
\text { saline (PBS), pH 7.2 }\end{array}$ & $\begin{array}{c}\text { Borate buffered saline } \\
\text { (BBS), pH 9.2, BSA }\end{array}$ \\
\hline HA titer & $<2$ & 2048 \\
\hline
\end{tabular}

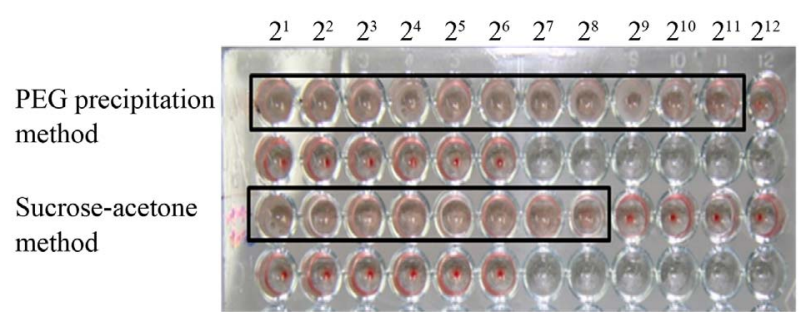

Figure 1. Comparison between two JEV antigens by the HA test. Each JEV antigens was diluted by serially two folds, and the HA titer was expressed as the reciprocal of the highest dilution of antigen showing complete hemagglutination. JEV antigen produced by PEG method represents 2048 HA titer and JEV antigen produced by sucrose acetone method showed 256 HA titer.
Disease Control and Prevention between 2007 and 2010 [2]. On the other hand, veterinary authorities carried out JEV serosurveillance in domestic animals including swine, goats, cattle, horses and wild migrating birds. Especially, wild birds captured in South Korea showed 86.7\% seropositive rate between 2007 and 2009 indicating that most wild birds captured in South Korea have been exposed to JEV [12]. Therefore, it is necessary to strengthen sero-survey of JE in several kinds of livestock.

In this study, we developed a new method to produce JEV antigen without using suckling mice. During the process of manufacturing JEV antigen, it is needed to inactivate the antigen. Beta propiolactone, acetylethlemine, formaldehyde and BEI have been used to inactivate several kinds of virus in preparing inactivated vaccines $[13,14]$. The BEI acts on the nucleic acid of virus and do not effect on the immunogenic properties of the viral protein. In contrast, formaldehyde acts on the surface protein of virus or bacteria and fixes the envelop protein of JEV that plays a key role in agglutinating goose erythrocytes and immunogenicity, tissue tropism, cell fusion, infection and maturation [15]. Therefore, the JEV antigen inactivated with $0.3 \%$ formaldehyde did not react on hemagglutination.

Many kinds of vector-borne viruses can agglutinate a broad range of animal species of erythrocytes, but, exact experimental $\mathrm{pH}$ condition is required for each virus [16]. When the optimal $\mathrm{pH}$ for the JEV is deduced, further HA titer will be carried out at the appropriate $\mathrm{pH}$ [17]. In this study, BEI inactivated antigen suspended in PBS at $\mathrm{pH}$ 7.2 did not agglutinate goose erythrocytes, but the antigen suspended in PBS at pH 9.0 containing bovine albumin revealed high HA activity with erythrocytes, indicating that optimum buffer condition such as $\mathrm{pH}$ or BSA should be applied for HA against JEV.

Sucrose acetone method using suckling mice has been used to produce JEV antigen but this method provokes animal welfare and environment contamination indicating that new method should be developed to replace the method. In this study, HA titer of JEV antigen produced by sucrose acetone method was found to be 256 . Generally, the virus produced in newborn mice yields high titer, but mouse brain material should be removed with several treatments such as acetone $[18,19]$. The reason why the antigen produced by sucrose acetone method show moderate high HA activity may be assumed that the antigen was extracted from brain homogenates inappropriately or might be lost in the process of producing JEV antigen.

In conclusion, the present results suggested that BEI inactivation method does not require treatment with acetone and can thus reduce environmental contamination with solvent. In addition, the JEV antigen inactivated by BEI can reduce public health risks in carrying out $\mathrm{HI}$ test. 


\section{Acknowledgements}

This work was financially supported by a grant (BAD142011-12-02) from the Animal and Plant and Fisheries Quarantine and Inspection Agency (QIA), Ministry for Food, Agriculture, Forestry and Fisheries (MIFAFF), Korea.

\section{REFERENCES}

[1] D. Harley, P. Bi, G. Hall, A. Swaminathan, S. Tong and C. Williams, "Climate Change and Infectious Diseases in Australia: Future Prospects, Adaptation Options, and Research Priorities," Asia-Pacific Journal of Public Health, Vol. 23, No. 2, 2011, pp. 54-66.

[2] D. K. Yang, Y. I. Oh, H. R. Kim, Y. J. Lee, O. K. Moon, H. Yoon, B. Kim, K. W. Lee and J. Y. Song, "Serosurveillance for Japanese Encephalitis Virus in Wild Birds Captured in Korea," Journal of Veterinary Science, Vol. 12, No. 4, 2011, pp. 373-377. doi:10.4142/jvs.2011.12.4.373

[3] G. L. Campbell, S. L. Hills, M. Fischer, J. A. Jacobson, C. H. Hoke, J. M. Hombach, A. A. Marfin, T. Solomon, T. F. Tsai, V. D. Tsu and A. S. Ginsburg, "Estimated Global Incidence of Japanese Encephalitis: A Systematic Review," Bull World Health Organization, Vol. 89, No. 10, 2011, pp. 766-774, doi:10.2471/BLT.10.085233

[4] Q. S. Cao, X. M. Li, Q. Y. Zhu, D. D. Wang, H. C. Chen and P. Qian, "Isolation and Molecular Characterization of Genotype 1 Japanese Encephalitis Virus SX09S-01, from Pigs in China," Virology Journal, Vol. 8, No. 1, 2011, p. 472. doi:10.1186/1743-422X-8-472

[5] E. Konishi, Y. Sakai, Y. Kitai and A. Yamanaka, "Prevalence of Antibodies to Japanese Encephalitis Virus among Inhabitants in Java Island, Indonesia, with a Small Pig Population," American Journal of Tropical Medicine and Hygiene, Vol. 80, No. 5, 2009, pp. 856-861.

[6] E. Konishi, M. Shoda and T. Kondo, "Prevalence of Antibody to Japanese Encephalitis Virus Nonstructural 1 Protein among Racehorses in Japan: Indication of Natural Infection and Need for Continuous Vaccination," Vaccine, Vol. 22, No. 9-10, 2004, pp. 1097-1103. doi:10.1016/j.vaccine.2003.10.001

[7] O. P. Larghi and A. E. Nebel, "Rabies Virus Inactivation by Binary Ethylenimine: New Method for Inactivated Vaccine Production," Journal of Clinical Microbiology, Vol. 11, No. 2, 1980, pp. 120-122.

[8] R. Putnak, D. A. Barvir, J. M. Burrous, D. R. Dubois, V. M. D'Andrea, C. H. Hoke, J. C. Sadoff and K. H. Eckels, "Development of a Purified, Inactivated, Dengue-2 Virus Vaccine Prototype in Vero Cells: Immunogenicity and Protection in Mice and Rhesus Monkeys," Journal of Infectious Diseases, Vol. 174, No. 6, 1996, pp. 1176-1184. doi:10.1093/infdis/174.6.1176
[9] G. D. Lewis and T. G. Metcalf, "Polyethylene Glycol Precipitation for Recovery of Pathogenic Viruses, Including Hepatitis a Virus and Human Rotavirus, from Oyster, Water, and Sediment Samples," Applied and Environmental Microbiology, Vol. 54, No. 8, 1988, pp. 1983-1988.

[10] D. H. Clarke and J. Casals, "Techniques for Hemagglutination and Hemagglutination-Inhibition with Arthropod-Borne Viruses," American Journal of Tropical Medicine and Hygiene, Vol. 7, No. 5, 1958, pp. 561-573.

[11] D. K. Yang, B. H. Kim, C. H. Kweon, J. H. Kwon, S. I. Lim and H. R. Han, "Biophysical Characterization of Japanese Encephalitis Virus (KV1899) Isolated from Pigs in Korea," Journal of Veterinary Science, Vol. 5, No. 2, 2004, pp. 125-130.

[12] S. M. Yun, J. E. Cho, Y. R. Ju, S. Y. Kim, J. Ryou, M. G. Han, W. Y. Choi and Y. E. Jeong, "Molecular Epidemiology of Japanese Encephalitis Virus Circulating in South Korea, 1983-2005," Virology Journal, Vol. 7, No. 14, 2010, p. 127. doi:10.1186/1743-422X-7-127

[13] H. G. Bahnemann, "Inactivation of Viral Antigens for Vaccine Preparation with Particular Reference to the Application of Binary Ethylenimine," Vaccine, Vol. 8, No. 4, 1990, pp. 299-303. doi:10.1016/0264-410X(90)90083-X

[14] M. A. Martín-Acebes, A. Vázquez-Calvo, M. GonzálezMagaldi and F. Sobrino, "Foot-and-Mouth Disease Virus Particles Inactivated with Binary Ethylenimine Are Efficiently Internalized into Cultured Cells," Vaccine, Vol. 29, No. 52, 2011, pp. 9655-9562. doi:10.1016/j.vaccine.2011.10.031

[15] P. C. McMinn, "The Molecular Basis of Virulence of the Encephalitogenic Flaviviruses," Journal of General Virology, Vol. 78, No. 11, 1997, pp. 2711-2722.

[16] E. N. Meeusen, J. Walker, A. Peters, P. P. Pastoret and G. Jungersen, "Current Status of Veterinary Vaccines," Clinical Microbiology Reviews, Vol. 20, No. 3, 2007, pp. 489-510. doi:10.1128/CMR.00005-07

[17] A. Igarashi, K. Buei, N. Ueba, M. Yoshida, S. Ito, H. Nakamura, F. Sasao and K. Fukai, "Isolation of Viruses from Female Culex Tritaeniorhynchusin Aedes Albopictus Cell Cultures," American Journal of Tropical Medicine and Hygiene, Vol. 30, No. 2, 1981, pp. 449-460.

[18] M. Abe, K. Shiosaki, L. Hammar, K. Sonoda, L. Xing, S. Kuzuhara, Y. Kino and R. Holland Cheng, "Immunological Equivalence between Mouse Brain-Derived and Vero Cell-Derived Japanese Encephalitis Vaccines," Virus Research, Vol. 121, No. 2, 2006, pp. 152-160. doi:10.1016/j.virusres.2006.05.004

[19] G. J. Chang, G. Kuno, D. E. Purdy and B. S. Davis, "Recent Advancement in Flavivirus Vaccine Development," Expert Reviews of Vaccines, Vol. 3, No. 2, 2004, pp. 199220. doi:10.1586/14760584.3.2.199 